\title{
Modelagem de uma Ferramenta de Suporte a OAM para Redes Metro Ethernet
}

\author{
Kleydson Wilbert M. Cunha e Sergio V. Fialho
}

\begin{abstract}
Resumo - Este artigo apresenta parte do desenvolvimento de uma ferramenta que dá suporte às atividades de Operação, Manutenção e Administração (OAM) de Redes Metropolitanas Ethernet, através da detecção de falhas e medição de desempenho da rede. A modelagem da ferramenta foi feita com base na proposta contida no padrão IEEE 802.3ah, chamada também de OAM EFM (Ethernet First Mile).

Palavras-Chave - Metro Ethernet, OAM, EFM.

Abstract - This article presents part of development of a tool that gives support to the activities of Operatiom, Maintenance and Managment (OAM) of Metropolitan Ethernet Networks, through fault detection and measurement of the network performance. The modeling was based on the IEEE 802.3ah standard, also called EFM (Ethernet Fist Mile) OAM.

Keywords - Metro Ethernet, OAM, EFM.
\end{abstract}

\section{INTRODUÇÃO}

As funções OAM (Operação, Administração e Manutenção) descrevem a instrumentação de redes de telecomunicações que provêem aos operadores as ferramentas para gerenciamento remoto de suas redes. No nível do dispositivo gerenciado, o protocolo OAM gera mensagens que são usadas pelos operadores, ajudandoos a identificar problemas que não sejam inicialmente aparentes. Por exemplo, o protocolo OAM permite monitoramento automático da rede, onde o operador recebe notificações dos equipamentos com falha. Em eventos de falha, a informação gerada pelo protocolo OAM ajuda ao operador a diagnosticar a rede para localizar a falha, identificando quais serviços foram prejudicados [1].

O Ethernet vem se transformando na tecnologia preferida de acesso banda-larga entre as redes das operadoras e dos usuários. Mas como seu uso em redes públicas continua a crescer, o Ethernet deve, como um protocolo de camada de nível 2, ser capaz de gerar informações sobre o comportamento da rede relativo à essa camada. As tecnologias predecessoras das operadoras de Telecomunicações, como SONET (Synchronous Optical Network) e ATM (Asynchronous Transfer Mode) já possuíam esta habilidade. Como esses protocolos ainda continuam sendo muito usados nos ambientes das operadoras, as atividades de OAM definidas para o Ethernet devem estar cientes dessas tecnologias legadas e estar aptas a coexistir com suas soluções de gerenciamento.

Com o lançamento das redes Ethernet metropolitanas (MAN Ethernet) e o advento da classe operadora Ethernet, surge a necessidade de gerenciar essas redes e, para que sejam realmente classe operadora, é essencial que suportem detecção automática de falhas e avaliação de desempenho. A fim de permitir aos provedores de serviço Ethernet operar e manter suas redes há uma necessidade de incluir OAM na camada Ethernet.

Kleydson Wilbert Modesto Cunha (kleydson @ bolsistas.pop-rn.rnp.br), Bolsista na área Redes Metro Ethernet e Sergio V. Fialho (fialho@pop-rn.rnp.br), coordenador da Rede GigaNatal, ambos do Departamento de Engenharia de Computação e Automação, Centro de Tecnologia, Universidade Federal do Rio Grande do Norte, Brasil.
Tardiamente surgiram dois protocolos OAM para Ethernet. Um deles foi desenvolvido para aplicações "Ethernet na primeira milha" (EFM), operando no nível de um enlace único, enquanto o outro atende ao problema mais amplo da conectividade Ethernet fim-a-fim e garantia de serviço. O OAM para camada de enlace foi desenvolvido pela força-tarefa IEEE 802.ah e por esta razão é conhecido como OAM "EFM" ou "802.3ah"[2]. No IEEE (Institute of Electrical and Electronics Engineers) o trabalho de especificação de OAM para a camada de serviço está sendo desenvolvido sob a responsabilidade da força tarefa 802.1ag (Gerenciamento de falhas na conectividade), enquanto na ITU-T (International Telecommunication Union) a recomendação está em fase de rascunho e foi chamada de Y.17ethoam(Y.1731).

Mais recentemente, o Metro Ethernet Fórum publicou uma nova especificação definindo o arcabouço e as exigências do Serviço OAM para redes metropolitanas Ethernet, a MEF 17 [3]. Esse documento não define nenhum protocolo específico a ser usado nas atividades de OAM, restringido-se a especificação dos conceitos relativos a Domínio, Componentes e Exigências OAM.

Apesar da evidente necessidade de ferramentas de suporte às atividades OAM para redes Metro Ethernet, ainda não se encontra no mercado nenhuma ferramenta OAM para essas redes. Assim, o objetivo desse trabalho é propor o desenvolvimento de uma ferramenta que seja instalada tanto na entidade local (local de gerenciamento da rede) quanto nas entidades remotas a serem gerenciadas. Como os padrões 802.1ag do IEEE e a recomendação da ITU-T Y.1731 ainda estão fase de padronização, a modelagem da ferramenta foi baseada a partir da documentação do IEEE 802.3ah. Essa ferramenta deve ser muito útil no suporte à operação da Rede GigaNatal. Essa é uma rede metropolitana acadêmica, que está sendo instalada em Natal, dentro do contexto do projeto REDECOMEP[4], coordenado pela Rede Nacional de Pesquisa. A figura 1 mostra a estratégia de instalação da ferramenta OAM projetada.

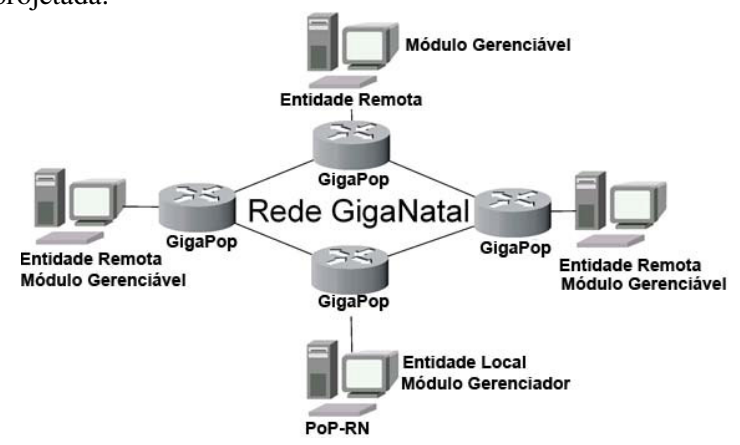

Figura 1 - Estratégia de instalação da ferramenta OAM

\section{DESCRIÇÃO DO FUNCIONAMENTO DA FERRAMENTA}

A ferramenta deve enviar mensagens OAM da camada de enlace, encapsuladas em quadros de protocolo lento sem 
identificação, chamados de OAMPDU (Unidade de Dados do Protocolo OAM). Essas mensagens são usadas para controlar as características operacionais do dispositivo Ethernet. Esses quadros são ditos lentos, devido à limitação com relação à sua taxa de envio (10 quadros por segundo), de forma a não sobrecarregar a rede. Os OAMPDU são transmitidos em modo multicast para um endereço multicast específico, com limitação de enlace. Isso significa que esses quadros só cruzam um único enlace, e nunca são encaminhados por "bridges" ou "switches", mesmo que implementem essas funcionalidades OAM.

As OAMPDU contêm as informações de controle e estado necessárias para monitorar, testar e solucionar problemas em enlaces com suporte à OAM. Essas informações são codificadas usando um código principal, acompanhadas das informações codificadas no formato TLV-type-lenght-value, conforme mostrado na figura 2.[5]

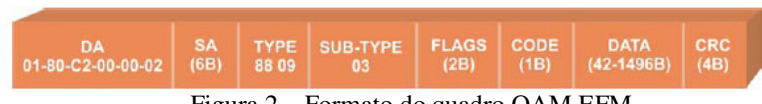

Figura 2 - Formato do quadro OAM EFM

Como nos dias atuais os equipamentos de rede ainda não estão equipados com essa tecnologia OAM, faz-se necessário a criação de dois módulos: um a ser instalado na entidade local, ou seja, a entidade responsável pela Operação, Administração e Manutenção da rede. Esse módulo conta com mais funcionalidades, pois o operador (administrador da rede) deve dispor de uma interface gráfica para poder usar todas as funcionalidades que o protocolo oferece. Um outro módulo deve ser instalado nas entidades remotas, para que estas, ainda sem capacidades OAM, sejam capazes de ler e processar quadros OAM, bem como responder aos pedidos gerados pela entidade local. Este módulo deve somente processar os quadros OAMPDU, não havendo necessidade de incluir uma interface para operador humano.

A ferramenta deve ter as seguintes funcionalidades:

- Descobrimento: Primeira fase, quando se identifica os dispositivos da rede, verificando suas capacidades OAM.

- Monitoramento de Enlace: Usado para detecção e indicação de falha nos enlaces, sob circunstâncias variadas.

- Indicação de Falha Remota: Permite a detecção das condições de falha no equipamento par:

$$
\begin{array}{ll}
\circ & \text { Falha no Enlace } \\
\circ & \text { Último Suspiro ("Dying Gasp”) } \\
\circ & \text { Evento Crítico }
\end{array}
$$

- "Loopback" Remoto: Ajuda a assegurar a qualidade dos enlaces durante a sua instalação ou durante a localização de erros.

- Recuperação de Variável MIB: Provê acesso remoto de somente leitura à variável MIB

\section{DESENVOLVIMENTO DA FERRAMENTA}

A partir da descrição informal apresentada na seção anterior, iniciou-se o desenvolvimento da ferramenta, através de sua especificação formal. Para isso utilizou-se a linguagem UML. A figura 3 mostra o diagrama de casos de uso da ferramenta, que reflete as funcionalidades descritas anteriormente.

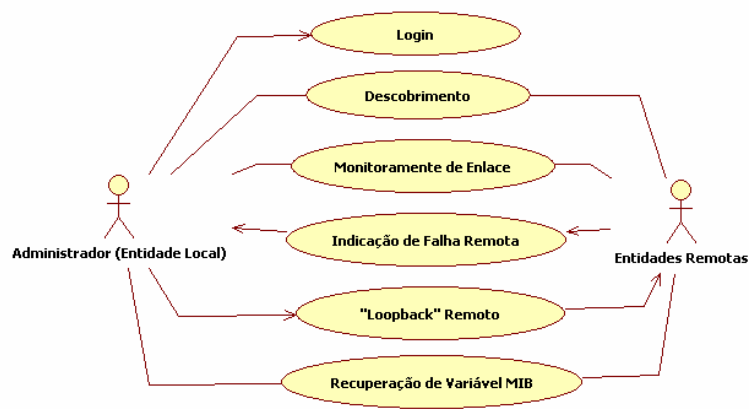

Figura 3 - Caso de Uso da Ferramenta

Os diagramas UML de classes e de seqüências já foram gerados e detalham cada um dos casos de uso mostrados na figura.

O módulo a ser instalado em uma Estação Remota necessita acessar recursos da máquina em baixo nível, uma vez que se destina a executar as funcionalidades OAM, comunicando-se exclusivamente com a Estação Local. Essa última, entretanto, além de necessitar um tipo de acesso análogo ao da Estação Remota, também deve permitir comunicação com um usuário humano, o Administrador, de preferência através de uma interface com recursos gráficos. Para a implementação dos módulos que necessitam realizar acessos de baixo nível aos recursos das máquinas escolheu-se o uso da linguagem C. Foi usada a tecnologia "Socket Raw", que permite a criação de quadros Ethernet, a princípio para transportar datagramas em forma bruta, através de um socket, mas que admite o envio de qualquer outro tipo de dado. O "Socket Raw" permite que se informe diretamente os endereços MAC de destino e de origem, o tipo do protocolo (no caso o valor hexadecimal 8809 , informando o uso de um protocolo lento, específico para OAM) e também o valor do campo de dados, contendo os valores dos outros campos especificados pelo protocolo IEEE 802.3ah, tais como os campos de: sub-tipo, flags, código e outros. Foram realizados alguns testes iniciais, quando se verificou o sucesso no envio de quadros através do mecanismo mencionado, recomendando seu uso mais extensivo na implementação da ferramenta.

\section{CONCLUSÕES}

O desenvolvimento de uma ferramenta para dar suporte às atividades OAM de uma Rede Metro Ethernet vem preencher uma lacuna existente e pode ser de grande valia na operação da Rede GigaNatal. Entretanto, até o momento como só existe o padrão IEEE 802.3ah para monitoramento dos enlaces Ethernet, adotou-se esse padrão como base para o desenvolvimento da ferramenta, tomando-se o cuidado de também manter a compatibilidade com os aspectos definidos na MEF 17. A especificação formal da ferramenta OAM está concluída e sua implementação se encontra em fase de desenvolvimento. Espera-se concluir essa implementação concomitantemente à entrada em operação da Rede GigaNatal, que deve ocorrer durante o presente semestre.

\section{REFERENCIAS}

[1] INDOO, P., Nortel. Ethernet OAM. Disponivel em: http://www.iec.org/newsletter/march07_1/broadband_2.html

[2] Metro Ethernet Fórum - Ethernet First Mile Operation, Administration and Maintenance (OAM) - A Tutorial, Novembro de 2005. Disponível em: http://www.metroethernetforum.org

[3] Metro Ethernet Fórum - MEF 17 - Service OAM Framework and Requirements - Phase 1. Abril, 2007. Disponível em: http://www.metroethernetforum.org

[4] http://www.redecomep.rnp.br/

[5] Yaakov (Jonathan) Stein, Chief Scientist, 2006. RAD Data Communications. Disponível http://www.dspcsp.com/RAD/ethoam_wp.pdf 